\title{
Nuevos conceptos en feocromocitoma y paraganglioma en el 2017
}

\author{
Alejandro Román-González ${ }^{1,2}$
}

\author{
${ }^{1}$ Docente de cátedra. Endocrinología, Facultad de Medicina, \\ Universidad de Antioquia \\ ${ }^{2}$ Departamento de Endocrinología, Hospital Universitario San \\ Vicente Fundación.
}

Correspondencia: Dr. Alejandro Román-González. Dirección: Calle 64 No 51d-154, Oficina Endocrinología y Diabetes. Hospital Universitario San Vicente Fundación, Medellín, Colombia. Teléfono: 2635397.

Correo electrónico: alejoroman@gmail.com

Fecha de recepción: 12/10/2017

Fecha de aceptación: 18/10/2017

\section{Resumen}

E l feocromocitoma y el paraganglioma son tumores neuroendocrinos derivados de la cresta neural. Son tumores infrecuentes en la práctica clínica diaria. El feocromocitoma está localizado en la médula suprarrenal y el paraganglioma es un tumor localizado en los paraganglios del sistema nervioso autónomo, por ende, su localización es extraadrenal. Existen predictores clínicos de malignidad como el tamaño, la localización y las mutaciones en SHDB. La presencia de predictores clínicos y la supervivencia según el compromiso metastásico en aquellos casos con feocromocitoma/ paraganglioma maligno ha permitido por primera vez crear la clasificación TNM para esta enfermedad. Adicionalmente, la evaluación genética se ha establecido como el paradigma de manejo. Los resultados del análisis del genoma del feocromocitoma confirman una alta heredabilidad de esta enfermedad y descubren nuevos genes que pueden convertirse en objetivos terapéuticos para el futuro.

\section{Introducción}

El feocromocitoma y el paraganglioma simpático son tumores neuroendocrinos infrecuentes ${ }^{(1)}$. El feocromocitoma se origina en la médula suprarrenal y el paraganglioma en los ganglios simpáticos o parasimpáticos. Es una causa rara de hipertensión de origen endocrino y es un tumor con alta heredabilidad $^{(2)}$. En el año 2017 se presentaron dos eventos importantes en feocromocitoma: por primera vez se creó la cla- sificación $\mathrm{TNM}^{(3)}$ para este tumor en un proyecto coordinado por el Dr. Camilo Jiménez y se presentaron los resultados del proyecto del genoma del feocromocitoma ${ }^{(4)}$. En esta revisión de tema se presentan de manera sucinta estos dos eventos y se presentan a la comunidad médica las perspectivas futuras en el manejo del feocromocitoma maligno ${ }^{(5-9)}$.

\section{Clasificación TNM}

La clasificación del cáncer es basada en el tamaño del tumor primario $(\mathrm{T})$, el compromiso ganglionar $(\mathrm{N})$ y la presencia de metástasis (M). Esta clasificación es realizada por el American Joint Committee on Cancer (AJCC). Para el feocromocitoma y paraganglioma no existía una clasificación TNM hasta este año. La importancia de tener una clasificación TNM es que homogeniza el manejo de los pacientes e indica a los clínicos las diferentes categorías de pacientes, lo que permite tomar decisiones terapéuticas acorde con el pronóstico de la enfermedad. En el caso del feocromocitoma, los determinantes principales en esta nueva clasificación son el tamaño del tumor, si es un paraganglioma y la presencia y localización de las metástasis. En términos generales, un tumor mayor de $5 \mathrm{~cm}$ tiene peor supervivencia y más riesgo de malignidad que un tumor pequeño. Los paragangliomas también tienen peor supervivencia y mayores tasas de malignidad $^{(10)}$. La localización del tumor predice mejor la agresividad que el tamaño. Mientras que solo $3 \%$ de los feocromocitomas menores de $5 \mathrm{~cm}$ hacen metástasis, los paragangliomas menores de $5 \mathrm{~cm}$ hacen compromiso a distancia hasta en el $20 \%$ de los casos. Una debilidad de esta clasificación es que no tiene en cuenta los aspectos genéticos y es posible que las mutaciones en SDHB tengan un peor pronóstico como ha sido sugerido en algunos estudios ${ }^{(11)}$. Sin embargo, pacientes con enfermedad aparentemente esporádica pueden tener un comportamiento clínico muy agresivo. A futuro, según se defina con claridad el pronóstico y se valide la clasificación, se tendrá en cuenta el genotipo del tumor ${ }^{(5)}$. El tipo de compromiso metastásico también determina el pronóstico. Los pacientes con metástasis óseas solas tienen mejor pronóstico que aquellos con metástasis hepáticas o metástasis en varios órganos (tabla 1). 
Tabla 1. Clasificación TNM del feocromocitoma y paraganglioma maligno ${ }^{(3)}$

\begin{tabular}{|c|c|}
\hline $\begin{array}{l}\text { Tamaño del tumor } \\
\text { primario }\end{array}$ & \\
\hline TX & Tamaño desconocido o no evaluable \\
\hline T1 & $\begin{array}{l}\text { Tumor }<5 \mathrm{~cm} \text { en la dimensión mayor, } \\
\text { sin invasión extraadrenal }\end{array}$ \\
\hline $\mathrm{T} 2$ & $\begin{array}{l}\text { Tumor } \geq 5 \mathrm{~cm} \text { o paraganglioma sim- } \\
\text { pático de cualquier tamaño sin inva- } \\
\text { sión extraadrenal }\end{array}$ \\
\hline כו & $\begin{array}{l}\text { Tumor de cualquier tamaño con com- } \\
\text { promiso en órganos cercanos como } \\
\text { hígado, páncreas, bazo y riñones }\end{array}$ \\
\hline Ganglios regionales & \\
\hline NX & Ganglios linfáticos no evaluables \\
\hline NO & Sin compromiso ganglionar \\
\hline N1 & $\begin{array}{l}\text { Metástasis en ganglios linfáticos re- } \\
\text { gionales }\end{array}$ \\
\hline Metástasis distantes & Sin metástasis \\
\hline Mo & Metástasis distantes \\
\hline M1 & M1a: Metástasis solo al hueso \\
\hline & $\begin{array}{l}\text { M1b: Metástasis distantes solo a gan- } \\
\text { glios linfáticos, hígado o pulmón }\end{array}$ \\
\hline & $\begin{array}{l}\text { M1c: Metástasis al hueso y otros ór- } \\
\text { ganos }\end{array}$ \\
\hline Estadios & \\
\hline Estadio 1 & T1 No M0 \\
\hline Estadio 2 & T2 NO MO \\
\hline Estadio 3 & $\mathrm{~T} 1 \quad \mathrm{~N} 1 \quad \mathrm{M} 0$ \\
\hline & T2 N1 $\mathrm{M} 0$ \\
\hline & T3 Cualquier N M0 \\
\hline Estadio 4 & Cualquier T Cualquier N M1 \\
\hline
\end{tabular}

\section{Estudios genéticos: Proyecto The Cancer Genome Atlas (TCGA)}

El feocromocitoma y el paraganglioma simpático se han asociado clásicamente a síndromes genéticos como la neoplasia endocrina múltiple tipo II ${ }^{(12-14)}$, el síndrome de Von Hippel-Lindau $^{(15,16)}$ y la neurofibromatosis ${ }^{(17)}$. Posteriormente, se describió que mutaciones en el complejo de la succinato deshidrogenasa generaban este tumor ${ }^{(18,19)}$. Este paso confirmó, por primera vez, que una alteración en una vía metabólica podía generar cáncer (figura 1). Posteriormente, se encontró que otros genes pueden asociarse a feocromocitoma, como por ejemplo FH, MAX, TMEM127, EPAS, MDH2, HIF2, ATRX, KIF1b, HRAS, MEN1, EGLN1, EGLN2 e IDH1 ${ }^{(20,21)}$. Dada la alta carga genética del feocromocitoma, este tumor fue incluido en el TCGA y sus resultados fueron presentados este año en la revis- ta $\mathrm{Cell}^{(4)}$. Se tomaron 173 tumores, 11 pacientes con metástasis y un total de 16 tumores agresivos, definidos por recurrencia o invasión local a ganglios. Se realizó en estos tumores secuenciación de $\mathrm{ADN}$, número de copias de $\mathrm{ADN}$, secuenciamiento de ARN y miARN, metilación de ADN y ensayos de proteínas en fase reversa. De los pacientes, $27 \%$ tenían mutaciones germinales. La mutación germinal más frecuente fue en el gen SHDB (9\%), seguido por RET (6\%), VHL (4\%) y NF1 (3\%). Se identificaron también mutaciones somáticas en CSDE1 y se encontraron genes fusionados en MAML2, BRAF, NGFR y NF1. Este estudio sugiere clasificar el feocromocitoma y paraganglioma en cuatro grupos moleculares definidos así: a. subtipo asociado a señalización por cinasas, b. subtipo asociado a pseudohipoxia, c. subtipo asociado a alteraciones en Wnt y d. subtipo asociado a mezcla cortical. La importancia de este estudio radica en que podemos conocer las bases moleculares de esta neoplasia y poder establecer objetivos terapéuticos, en particular para el manejo del feocromocitoma y paraganglioma metastásicos. En nuestro medio, es poco posible acceder a estudios de secuenciación genética múltiple y el estudio para gen único toma tiempo para ser autorizado y realizado. Estudios preliminares en nuestro medio realizados por el grupo de la Universidad de Antioquia, el Hospital Universitario San Vicente Fundación y el Hospital Pablo Tobón Uribe sugieren que RET es la mutación más frecuente. Sin embargo, estos datos están sesgados por la búsqueda activa de los familiares de los sujetos con neoplasia endocrina múltiple tipo II por nuestro grupo. La segunda mutación más frecuente fue en el gen SDHB.

\section{Estudios actuales y futuros en feocromocitoma y paraganglioma metastásico}

A futuro se presentarán resultados de tres agentes terapéuticos que están en evaluación para estos pacientes que actualmente tienen opciones terapéuticas limitadas y ninguna con aprobación por los agentes regulatorios.

\section{Sunitinib}

Este medicamento es un inhibidor multicinasa del VEGFR-1, VEGFR-2, PDGFB receptor, RET y c-KIT ${ }^{(22,23)}$. Sunitinib tiene actividad antitumoral y antiangiogénica y es un medicamento aprobado para el cáncer renal, los tumores neuroendocrinos pancreáticos y los tumores estromales gastrointestinales ${ }^{(24)}$. El estudio más grande fue realizado por dos centros de referencia de Europa y Estados Unidos ${ }^{(25)}$. De 17 pacientes con feocromocitoma maligno en progresión incluidos en el estudio, 50\% tenían mutaciones en la SDHB, la mayoría tenía enfermedad sin respuesta a quimioterapia con CVD y/o MIBG. En estos pacientes, el $47 \%$ tenía alguna respuesta acorde a los criterios de RECIST $1.1^{(26)}$. Además, los pacientes con respuesta que fueron evaluados por PET-CT con ${ }^{18} \mathrm{~F}$-FDG demostraron una reducción 
Figura 1. Complejo de la succinato deshidrogenasa

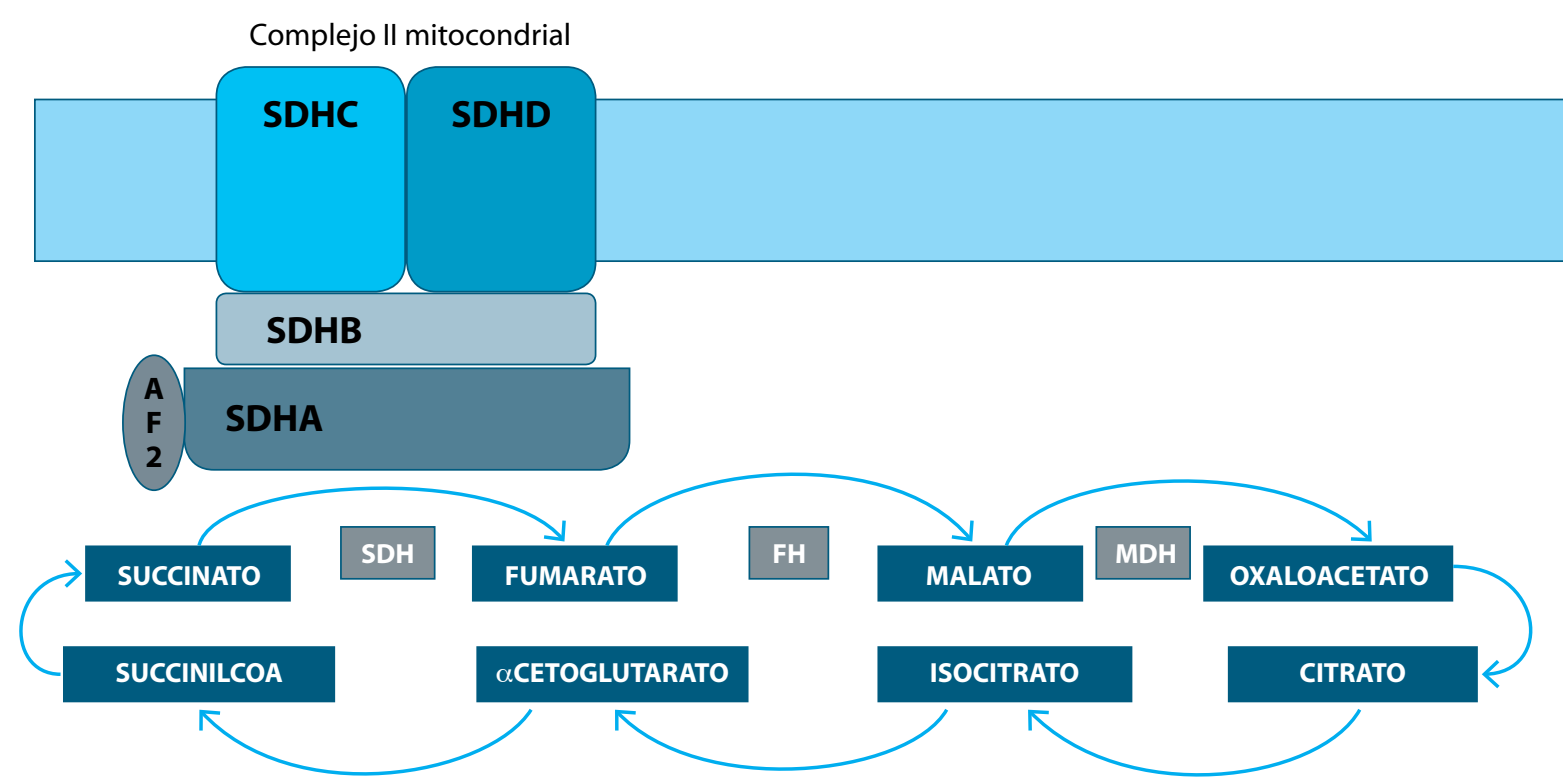

Figura 2. Opciones actuales y futuras para el feocromocitoma y paraganglioma con enfermedad metastásica

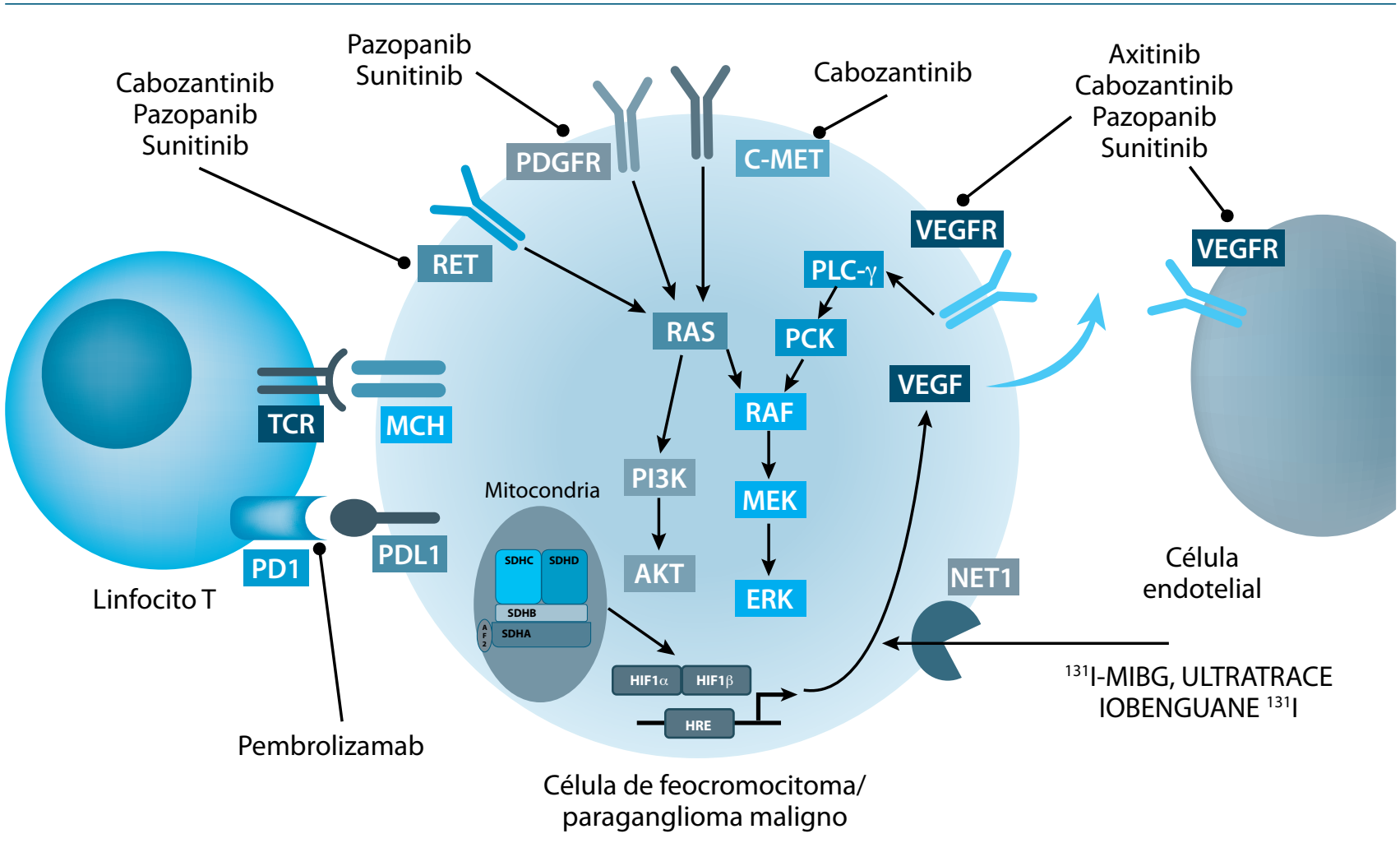

en la captación de glucosa. Hubo mejoría en la hipertensión y en la dosis de medicamentos que usaban para la presión arterial. La supervivencia total y la supervivencia libre de enfermedad fue de 26,7 y 4 meses respectivamente. La respuesta más larga documentada fue 36 meses. Este estudio sugiere que sunitinib puede beneficiar algunos pacientes con feocromocitoma o paraganglioma maligno en progresión, incluso en pacientes con resistencia a la quimioterapia o MIBG independiente de las mutaciones en SHDB. Previo al uso de esta terapia debe controlarse apropiadamente el dolor y la hipertensión. 
En camino se están realizando dos estudios fase II en pacientes con esta enfermedad. El primero es el SNIPP (Sunitinib in Patients with Recurrent Paraganglioma/Pheochromocytoma). En este estudio se evaluará sunitinib 50 mg al día durante cuatro semanas y descanso de dos semanas. Aún está en fase de reclutamiento. El desenlace primario es la mejor respuesta clínica acorde al RECIST. El segundo estudio es el FIRSTMAPPP (The First International Randomized Study in Malignant Progressive Pheochromocytoma and Paraganglioma). La dosis en estudio de sunitinib es 37,5 mg cada día y se evaluará la supervivencia libre de progresión. Este estudio ya finalizó el reclutamiento de los pacientes.

\section{Cabozantinib}

Es un inhibidor multicinasa de VEGFR1, VEGFR2, RET y c$M^{2} T^{(27,28)}$. Está aprobado para el tratamiento del cáncer medular de tiroides y el carcinoma renal. Es un inhibidor potente de la angiogénesis. Algunos estudios sugieren que cabozantinib afecta el microambiente óseo y puede tener mejores desenlaces en pacientes con compromiso metastásico óseo, un evento frecuente en feocromocitoma maligno. Actualmente está siendo evaluado en un estudio fase II prospectivo para el tratamiento de feocromocitoma y paraganglioma (NCT02302833). El desenlace primario es la tasa de respuesta total. La dosis es $60 \mathrm{mg}$ cada 24 horas y algunos reportes preliminares de este estudio han mostrado respuesta o estabilidad de la enfermedad en 10 de 11 pacientes sin efectos adversos graves ${ }^{(8)}$.

\section{Iogenbuano Ultratrace $I^{131}$}

Una nueva terapia en estudio es Ultratrace iobenguano ${ }^{131} \mathrm{I}$. Esta es una forma de ${ }^{131}$ I-MIBG que tiene una actividad altamente específica ${ }^{(29)}$. Ultratrace iobenguano ${ }^{131}$ I ha sido usada seguramente en humanos ${ }^{(30)}$ y se está usando en estudios fase II para evaluar su actividad. El desenlace primario es la reducción en los antihipertensivos con objetivos secundarios como respuesta tumoral, seguridad, calidad de vida y supervivencia ${ }^{(31)}$. Los resultados preliminares iniciales con 49 pacientes han demostrado que 16 (32\%) alcanzan una reducción del 50\% de los antihipertensivos y que 17 (35\%) tienen una respuesta parcial acorde a RECIST. La mediana de supervivencia fue 36 meses $^{(9)}$. El estudio ya terminó reclutamiento y la compañía Progenics ha manifestado que el estudio tuvo resultados positivos. El artículo final no ha sido publicado a la fecha de redacción de este manuscrito, pero los datos disponibles demuestran que se logró el desenlace primario y que se logró una disminución en el tamaño tumoral en el $85 \%$ de los pacientes. Estos datos fueron sometidos a la FDA y es muy posible que este radio-fármaco se convierta en la primera terapia aprobada para el feocromocitoma y paraganglioma maligno y por ende en la primera línea de tratamiento en aquellos pacientes con enfermedad progresiva con captación en una gammagrafía con MIBG.

\section{Aspectos quirúrgicos del feocromocitoma maligno: nuevos hallazgos}

El tratamiento de la enfermedad metastásica ha sido clásicamente paliativo, dado que a la fecha no contamos con terapias curativas. En algunos tumores neuroendocrinos y otros tipos de cáncer se ha encontrado que la resección del tumor primario puede mejorar la supervivencia ${ }^{(32-34)}$. Un estudio retrospectivo de la base de datos del Surveillance, Epidemiology, and End Results Program sugiere que la ausencia de resección de cualquier carga tumoral puede ser un factor de riesgo para mayor mortalidad ${ }^{(35)}$. La resección del tumor primario en feocromocitoma puede disminuir la carga tumoral, lo que permite que las terapias sistémicas tengan mejores desenlaces, previene las complicaciones locales por extensión de la enfermedad, disminuir los síntomas catecolaminérgicos y sus complicaciones y, finalmente, puede mejorar la supervivencia. En este sentido, se han publicado dos trabajos importantes. El primero fue publicado por el grupo de la Mayo Clinic. Es un estudio retrospectivo con una muestra pequeña de pacientes donde se demostró una mejoría en las concentraciones de catecolaminas, mejorías en los síntomas catecolaminérgicos, reducción en los medicamentos antihipertensivos y extensión en la supervivencia ${ }^{(36)}$. El segundo estudio es, a la fecha de este manuscrito, el estudio más grande con resección del tumor primario. Fue realizado en el MD Anderson Cancer Center y el objetivo primario fue determinar la mejoría en supervivencia con resección del tumor primario ${ }^{(6)}$. Se evaluó la base de datos de feocromocitoma y paraganglioma maligno entre enero del 2000 y enero 2015. Se identificaron 113 pacientes. Los pacientes en quienes se resecó el tumor primario tuvieron una mejor supervivencia, comparados con aquellos en quienes no se realizó resección (148 meses, intervalo de confianza 95\% [IC] $112,8-183,2$ vs. 36 meses, 95\% IC 27,2-44,8; p<0,001). Se analizó un subgrupo con enfermedad sincrónica $(n=53)$ y en este grupo se encontró también una mejoría en la supervivencia. El análisis multivariado y un análisis de propensión confirmó estos hallazgos. Los síntomas de excesos catecolaminérgicos también mejoraron.

Estos dos estudios sugieren entonces que la resección del tumor primario en enfermedad metastásica es posible, que mejora los síntomas catecolaminérgicos y puede prolongar la supervivencia. 


\section{Referencias}

1. Román-González A, Sierra-Zuluaga J, Gutiérrez-Restrepo J, Builes-Barrera C, Jiménez-Vásquez C. Feocromocitoma-Paraganglioma: revisión de tema. Medicina y Laboratorio. 2015;21(5-6):111-30.

2. Dahia PL. Pheochromocytoma and paraganglioma pathogenesis: learning from genetic heterogeneity. Nat Rev Cancer. 2014;14(2):108-19.

3. Jimenez C, Libutti S, Landry C, Lloyd R, McKay R, Rohren E, et al. AdrenalNeuroendocrine Tumors. In: Amin MB, Edge S, Greene F, Byrd DR, Brookland RK, Washington MK, et al., editors. AJCC Cancer Staging Manual. 8 ed. New York: Springer; 2017. p. 919-27.

4. Fishbein L, Leshchiner I, Walter V, Danilova L, Robertson AG, Johnson AR, et al. Comprehensive Molecular Characterization of Pheochromocytoma and Paraganglioma. Cancer Cell. 2017.

5. Roman-Gonzalez A, Jimenez C. Malignant pheochromocytoma-paraganglioma: pathogenesis, TNM staging, and current clinical trials. Curr Opin Endocrinol Diabetes Obes. 2017.

6. Roman-Gonzalez A, Zhou S, Ayala-Ramirez M, Shen C, Waguespack SG, Habra MA, et al. Impact of Surgical Resection of the Primary Tumor on Overall Survival in Patients With Metastatic Pheochromocytoma or Sympathetic Paraganglioma. Ann Surg. 2017.

7. Roman-González A, Jiménez-Vásquez P, Hyde SM, Jessop A, Jimenez C. Management of Locally Advanced and Metastatic Pheochromocytoma and Paraganglioma. In: Electron K, editor. Management of Adrenal Masses in Children and Adults. 1: Springer International Publishing; 2016. p. 277-300.

8. Jimenez C, Waguespack S, Habra M, Busaidy N, Dadu R, Tamsen G, et al. Cabozantanib in patients with unresectable metastatic pheochromocytoma and paraganglioma. Global Academic Programs of Cancer Centers Symposium; Houston, Tx2017.

9. Jimenez C, Pryma DA, Sullivan DC, Schwarz JK, Noto RB, Stambler N, et al. Long Term Follow-up of a Pivotal Phase 2 Study of Ultratrace ${ }^{\circledR}$ Iobenguane I-131 (AZEDRA ${ }^{\mathrm{TM}}$ ) in Patients with Malignant Relapsed/Refractory Pheochromocytoma (Pheo)/Paraganglioma (Para). Endocrine Reviews. 2015;36(2):OR24-6.

10. Ayala-Ramirez M, Feng L, Johnson MM, Ejaz S, Habra MA, Rich T, et al. Clinical risk factors for malignancy and overall survival in patients with pheochromocytomas and sympathetic paragangliomas: primary tumor size and primary tumor location as prognostic indicators. J Clin Endocrinol Metab. 2011;96(3):717-25.

11. Amar L, Baudin E, Burnichon N, Peyrard S, Silvera S, Bertherat J, et al. Succinate dehydrogenase $B$ gene mutations predict survival in patients with malignant pheochromocytomas or paragangliomas. J Clin Endocrinol Metab. 2007;92(10):3822-8.

12. Waguespack SG, Rich T, Grubbs E, Ying AK, Perrier ND, Ayala-Ramirez M, et al. A current review of the etiology, diagnosis, and treatment of pediatric pheochromocytoma and paraganglioma. J Clin Endocrinol Metab. 2010;95(5):2023-37.

13. Cascón A, Pita G, Burnichon N, Landa I, López-Jiménez E, Montero-Conde $\mathrm{C}$, et al. Genetics of pheochromocytoma and paraganglioma in Spanish patients. J Clin Endocrinol Metab. 2009;94(5):1701-5.

14. Thosani S, Ayala-Ramirez M, Palmer L, Hu MI, Rich T, Gagel RF, et al. The characterization of pheochromocytoma and its impact on overall survival in multiple endocrine neoplasia type 2. J Clin Endocrinol Metab. 2013;98(11):E1813-9.

15. Rich TA, Jonasch E, Matin S, Waguespack SG, Gombos DS, Santarpia L, et al. A novel von Hippel-Lindau point mutation presents as apparently sporadic pheochromocytoma. Cancer Invest. 2008;26(6):642-6.

16. Jimenez C, Cabanillas ME, Santarpia L, Jonasch E, Kyle KL, Lano EA, et al. Use of the tyrosine kinase inhibitor sunitinib in a patient with von HippelLindau disease: targeting angiogenic factors in pheochromocytoma and other von Hippel-Lindau disease-related tumors. J Clin Endocrinol Metab. 2009;94(2):386-91.

17. Gruber LM, Erickson D, Babovic-Vuksanovic D, Thompson GB, Young WF, Bancos I. Pheochromocytoma and paraganglioma in patients with neurofibromatosis type 1. Clin Endocrinol (Oxf). 2017;86(1):141-9.
18. Baysal BE, Ferrell RE, Willett-Brozick JE, Lawrence EC, Myssiorek D, Bosch A, et al. Mutations in SDHD, a mitochondrial complex II gene, in hereditary paraganglioma. Science. 2000;287(5454):848-51.

19. Baysal BE, Maher ER. 15 YEARS OF PARAGANGLIOMA: Genetics and mechanism of pheochromocytoma-paraganglioma syndromes characterized by germline SDHB and SDHD mutations. Endocr Relat Cancer. 2015;22(4):T7182.

20. Castro-Vega LJ, Lepoutre-Lussey C, Gimenez-Roqueplo AP, Favier J. Rethinking pheochromocytomas and paragangliomas from a genomic perspective. Oncogene. 2016;35(9):1080-9.

21. Pillai S, Gopalan V, Smith RA, Lam AK. Updates on the genetics and the clinical impacts on phaeochromocytoma and paraganglioma in the new era. Crit Rev Oncol Hematol. 2016;100:190-208.

22. Choueiri TK, Halabi S, Sanford BL, Hahn O, Michaelson MD, Walsh MK, et al. Cabozantinib Versus Sunitinib As Initial Targeted Therapy for Patients With Metastatic Renal Cell Carcinoma of Poor or Intermediate Risk: The Alliance A031203 CABOSUN Trial. Journal of Clinical Oncology 2016;Published online before print November 14, 2016

23. Hao Z, Sadek I. Sunitinib: the antiangiogenic effects and beyond. Onco Targets Ther. 2016;9:5495-505.

24. Jimenez C, Rohren E, Habra MA, Rich T, Jimenez P, Ayala-Ramirez M, et al. Current and future treatments for malignant pheochromocytoma and sympathetic paraganglioma. Curr Oncol Rep. 2013;15(4):356-71.

25. Baudin E, Habra MA, Deschamps F, Cote G, Dumont F, Cabanillas M, et al. Therapy of endocrine disease: treatment of malignant pheochromocytoma and paraganglioma. Eur J Endocrinol. 2014;171(3):R111-22.

26. Ayala-Ramirez M, Chougnet CN, Habra MA, Palmer JL, Leboulleux S, Cabanillas ME, et al. Treatment with sunitinib for patients with progressive metastatic pheochromocytomas and sympathetic paragangliomas. J Clin Endocrinol Metab. 2012;97(11):4040-50.

27. Yakes FM, Chen J, Tan J, Yamaguchi K, Shi Y, Yu P, et al. Cabozantinib (XL184), a novel MET and VEGFR2 inhibitor, simultaneously suppresses metastasis, angiogenesis, and tumor growth. Mol Cancer Ther. 2011;10(12):2298-308.

28. Gordon MS, Vogelzang NJ, Schoffski P, Daud A, Spira AI, O'Keeffe BA, et al. Activity of cabozantinib (XL184) in soft tissue and bone: Results of a phase II randomized discontinuation trial (RDT) in patients (pts) with advanced solid tumors. J Clin Oncol. 2011;29 (suppl; abstr 3010).

29. Barrett JA, Joyal JL, Hillier SM, Maresca KP, Femia FJ, Kronauge JF, et al. Comparison of high-specific-activity ultratrace 123/131I-MIBG and carrieradded 123/131I-MIBG on efficacy, pharmacokinetics, and tissue distribution. Cancer biotherapy \& radiopharmaceuticals. 2010;25(3):299-308.

30. Coleman RE, Stubbs JB, Barrett JA, de la Guardia M, Lafrance N, Babich JW Radiation dosimetry, pharmacokinetics, and safety of ultratrace Iobenguane I-131 in patients with malignant pheochromocytoma/paraganglioma or metastatic carcinoid. Cancer biotherapy \& radiopharmaceuticals. 2009;24(4):469-75.

31. https://clinicaltrials.gov/ct2/show/NCT00874614 [

32. Bertani E, Fazio N, Botteri E, Chiappa A, Falconi M, Grana C, et al. Resection of the primary pancreatic neuroendocrine tumor in patients with unresectable liver metastases: possible indications for a multimodal approach. Surgery. 2014;155(4):607-14.

33. Capurso G, Bettini R, Rinzivillo M, Boninsegna L, Delle Fave G, Falconi M. Role of resection of the primary pancreatic neuroendocrine tumour only in patients with unresectable metastatic liver disease: a systematic review. Neuroendocrinology. 2011;93(4):223-9.

34. Dy BM, Strajina V, Cayo AK, Richards ML, Farley DR, Grant CS, et al. Surgical resection of synchronously metastatic adrenocortical cancer. Ann Surg Oncol. 2015;22(1):146-51.

35. Goffredo P, Sosa JA, Roman SA. Malignant pheochromocytoma and paraganglioma: a population level analysis of long-term survival over two decades. J Surg Oncol. 2013;107(6):659-64.

36. Strajina V, Dy BM, Farley DR, Richards ML, McKenzie TJ, Bible KC, et al. Surgical Treatment of Malignant Pheochromocytoma and Paraganglioma: Retrospective Case Series. Ann Surg Oncol. 2017. 